\title{
An in vivo experimental model for effects of topical retinoic acid in human skin
}

\author{
C.E.M.GRIFFITHS, L.J.FINKEL, M.G.TRANFAGLIA, T.A.HAMILTON AND \\ J.J.VOORHEES \\ Dermatopharmacology Unit, Department of Dermatology. University of Michigan Medical Center, Ann Arbor, Michigan. U.S.A. \\ Accepted for publication 1 April 1993
}

\begin{abstract}
Summary An occlusive patch-test assay has been developed for assessment of topical retinoid action in human epidermis. Previous work with this assay has demonstrated marked epidermal hyperplasia in skin treated with topical all-trans-retinoic acid for 4 days and similar effects with the local irritant, sodium lauryl sulphate. To investigate the capabilities of this assay further, a time-course and dose-response were performed with all-trans-retinoic acid, and a comparison made with sodium lauryl sulphate.

At no time, between 1 and 4 days, could the clinical or histological effects of $0.1 \%$ and $0.025 \%$ cream formulations of all-trans-retinoic acid be distinguished from each other. Epidermal hyperplasia was used to generate a 4-day dose-response for all-trans-retinoic acid at concentrations from $0 \cdot 001$ to $0.025 \%$ dissolved in a $70 \%$ ethanol $/ 30 \%$ propylene glycol vehicle. All-trans-retinoic acid could be successfully differentiated from sodium lauryl sulphate at 2 days by virtue of its greater ability to increase epidermal thickness, spongiosis and glycosaminoglycan deposition.

It appears that although all-trans-retinoic acid and sodium lauryl sulphate produce similar epidermal histological changes at 4 days, significant differences at earlier time-points suggest differing mechanisms of action. In addition, this in vivo human assay is able to provide potency ranking for doses of all-trans-retinoic acid, and may predict clinical efficacy of retinoids in improvement of acne and/or photodamage.
\end{abstract}

Both in vitro and murine models have been used to predict the efficacy of retinoids in the treatment of acne and photodamage. ${ }^{1-6}$ However, these models are not truly representative of the human in vivo situation, as retinoids can produce effects in vitro which are paradoxical to those observed in vivo. ${ }^{7}$ Furthermore, pharmacological penetration of drugs is often greater in murine than human epidermis. We have developed a short-term, 4-day assay to study the epidermal effects of topical retinoids in normal human skin. ${ }^{8}$ In this assay, a single application, and subsequent occlusion, of $0 \cdot 1 \%$ all-transretinoic acid (RA) cream for 4 days produces consistent erythema and characteristic epidermal changes, i.e. epidermal hyperplasia, thickened granular layer, spongiosis, increased number of mitotic figures and deposition of hyaluronidase-digestable colloidal iron-positive glycosaminoglycans (GAG). ${ }^{8}$ We have found that without occlusion the reproducibility of the patch test is inconsistent. The observed light microscopic epidermal

Correspondence: Dr C.E.M.Griffiths, Department of Dermatology, University of Michigan Medical Center, 1910 Taubman Center, Ann Arbor, MI 48109-0314, U.S.A. changes in this 4-day assay are similar to those reported after treatment of photodamaged skin with RA cream (tretinoin) for up to 10 months. ${ }^{9-11}$ In contrast, these changes are not observed in vehicle-treated skin.

Topical RA is effective in improving certain clinical features of photodamaged skin, ${ }^{9,11-16}$ although the presence of an erythematous, desquamative retinoid reaction has prompted the argument that RA is acting purely as a local irritant. Indeed, previous work has demonstrated that an irritant, sodium lauryl sulphate (SLS), as a $2 \%$ formulation in Retin- $A^{\mathrm{TM}}$ vehicle cream, produces similar clinical and haematoxylin and eosin-detectable epidermal histological changes as does $0 \cdot 1 \% \mathrm{RA}$ in the 4-day assay. ${ }^{8}$ To date, the only histological difference in this assay is a significantly greater deposition of GAG in $0 \cdot 1 \%$ RA vs. SLS-treated epidermis. ${ }^{17}$ Consequently, it is of interest to determine whether other significant differences exist between RA and SLS.

In this study we have addressed two issues: does the use of a shorter time-course for the assay (i.e., less than 4 days) allow a clearer histological distinction between RA and SLS effects, and is the assay sensitive enough to generate a dose-response for RA? 


\section{Methods}

Normal volunteers, (all of whom had given written consent in a protocol approved by the University of Michigan Institutional Review Board) had the various test agents applied to buttock skin under 'Saran Wrap' occlusion for the designated periods of time, and in a blinded, randomized fashion. Approximately $150 \mathrm{mg}$ of cream or $50 \mu \mathrm{l}$ of ETOH/PG were applied to a $9-\mathrm{cm}^{2}$ area of skin. On removal of the occlusive patch the degree of clinical erythema was scored using a 0-9 scale: 0 , no erythema; $1-3$, mild; $4-6$, moderate; and 7-9, severe erythema. Punch biopsies of the treated areas were performed under $1 \%$ plain lidocaine (lignocaine) anaesthesia, and placed in $10 \%$ neutral-buffered formalin.

Ortho Pharmaceutical Corporation (Raritan, NJ, U.S.A.) supplied the $0 \cdot 1 \%$ and $0 \cdot 025 \%$ RA cream (Retin$\left.A^{\mathrm{TM}}\right)$, Retin- $\mathrm{A}^{\mathrm{TM}}$ vehicle, and RA in ETOH/PG vehicle. SLS was obtained from E.I.Dupont De Nemours \& Company (Wilmington, DE, U.S.A.) and compounded at $2 \%$ in Retin- $\mathrm{A}^{\mathrm{TM}}$ vehicle cream. The $2 \%$ SLS formulation was used, as this produces almost identical erythema and epidermal histology as $0 \cdot 1 \%$ RA in the 4 -day assay. ${ }^{7}$

\section{Histology}

Biopsies were paraffin-embedded, and sections stained with haematoxylin and eosin. Two-day biopsies were also stained with colloidal iron for assessment of GAG. The histology was read at the light microscope level by one dermatopathologist (L.J.F.), who was unaware of the clinical results and from which treatment group the specimens had originated. Stratum corneum compaction, spongiosis, and granular layer thickness were assessed using an ordinal $0-4$ scale: where $0=$ absence of the parameter, and $4=$ maximum expression of the parameter. Epidermal thickness measured in $\mu \mathrm{m}$ from the base of the stratum corneum to the basement membrane of the inter-rete ridges was assessed in five high-power fields (h.p.f.), and mean thickness used. Mitotic figures in the epidermis were counted per five h.p.f. (in the 4-day RA cream study, 10 h.p.f. were used), and the mean value used. Colloidal iron-positive GAG within the epidermis were quantified using the same $0-4$ scale as for the other parameters.

\section{Comparison of RA and vehicle creams at 4 days}

$0 \cdot 1 \%$ and $0 \cdot 025 \%$ RA cream and Retin- $A^{\mathrm{TM}}$ vehicle were occluded for 4 days $(n=10)$. Erythema and histology were assessed for each test agent.
Time-course and dose-response of RA and SLS creams

$0 \cdot 1 \%$ and $0 \cdot 025 \%$ RA cream, RA cream vehicle and $2 \%$ SLS cream were compared at 3 days $(n=7), 2$ days $(n=9)$ and 1 day $(n=4)$. Erythema and histology were assessed for each test agent.

\section{Dose-response of RA in ETOH/PG vehicle}

$0 \cdot 001 \%, 0 \cdot 0025 \%, 0 \cdot 005 \%, 0 \cdot 01 \%$ and $0.025 \%$ RA in ETOH/PG and ETOH/PG alone were compared both clinically and histologically at 4 days $(n=30)$. A direct comparison with SLS in ETOH/PG was not possible due to the low solubility of SLS in this vehicle.

\section{Statistical methods}

For each assay, treatment group comparisons were assessed with a repeated measures analysis of variance and the Tukey procedure for multiple comparisons. Twosided significance was determined at the $0 \cdot 05$ and $0 \cdot 01$ type I error rates.

Summary data are represented as means \pm SEM. The analyses were performed with the use of BMDP Statistical Software.

\section{Results}

\section{Comparison of $R A$ creams at 4 days}

There was no significant difference between the degree of erythema produced by $0.1 \%$ and $0.025 \%$ RA creams, the mean erythema values being $4 \cdot 3 \pm 0 \cdot 4$ and $4 \cdot 4 \pm 0 \cdot 6$, respectively. Both concentrations of RA produced significantly greater erythema than RA vehicle $(1 \cdot 8 \pm 0 \cdot 6$, Table 1$)$.

Histological examination revealed characteristic epidermal effects, consisting of epidermal hyperplasia, spongiosis, granular layer expansion and increased mitotic counts, for both $0 \cdot 1 \%$ and $0 \cdot 025 \%$ RA (Fig. 1 , Table 1 ). $0 \cdot 1 \%$ and $0 \cdot 025 \%$ RA creams were indistinguishable from each other.

\section{Time-course and dose-response of RA and $2 \%$ SLS creams}

Three days. There was no significant difference in erythema produced by $0 \cdot 1 \%$ RA, $0 \cdot 025 \%$ RA or $2 \%$ SLS, although all three agents produced significantly more erythema than did vehicle. Except for granular layer thickness, $0 \cdot 1 \%$ and $0 \cdot 025 \%$ RA produced significant histological changes compared with vehicle. For all histological parameters the two concentrations of RA 
Table 1. 4-day patch test: clinical and histological parameters for $0 \cdot 1 \%$ RA, $0 \cdot 025 \%$ $\mathrm{RA}$, and vehicle creams

Figure 1. Photomicrographs of skin treated for 4 days under occlusion with (a) vehicle cream: (b) $0 \cdot 1 \%$ RA cream and (c) $0.025 \%$ RA cream. Note increased epidermal thickness and granular cell layer in the RAtreated skin. The epidermal histology for $0 \cdot 1 \%$ and $0.025 \%$ RA creams is indistinguishable at 4 days. (Haematoxylin and eosin, original magnification $\times 50$ ).

Table 2. 3-day patch test: clinical and histological parameters for $0 \cdot 1 \%$ RA, $0 \cdot 025 \%$ RA, $2 \%$ SLS and vehicle creams

\begin{tabular}{lccc}
\hline & $0 \cdot 1 \% \mathrm{RA}$ & $0 \cdot 025 \% \mathrm{RA}$ & Vehicle \\
\hline Erythema & $4 \cdot 3 \pm 0 \cdot 4^{\mathrm{a}}$ & $4 \cdot 4 \pm 0 \cdot 6^{\mathrm{a}}$ & $1 \cdot 8 \pm 0 \cdot 6^{\mathrm{b}}$ \\
Stratum corneum compaction & $1 \cdot 8 \pm 0 \cdot 4^{\mathrm{a}}$ & $1 \cdot 9 \pm 0 \cdot 4^{\mathrm{a}}$ & $1 \cdot 7 \pm 0 \cdot 4^{\mathrm{a}}$ \\
Granular layer thickness & $2 \cdot 6 \pm 0 \cdot 2^{\mathrm{a}}$ & $2 \cdot 3 \pm 0 \cdot 4^{\mathrm{a}}$ & $1 \cdot 2 \pm 0 \cdot 1^{\mathrm{b}}$ \\
Spongiosis & $2 \cdot 2 \pm 0 \cdot 2^{\mathrm{a}}$ & $1 \cdot 8 \pm 0 \cdot 2^{\mathrm{ab}}$ & $1 \cdot 5 \pm 0 \cdot 3^{\mathrm{b}}$ \\
Mitoses/10 h.p.f. & $2 \cdot 8 \pm 0 \cdot 6^{\mathrm{a}}$ & $1 \cdot 6 \pm 0 \cdot 5^{\mathrm{ab}}$ & $0 \cdot 5 \pm 0 \cdot 2^{\mathrm{b}}$ \\
Epidermal thickness $(\mu \mathrm{m})$ & $93 \pm 6^{\mathrm{a}}$ & $84 \pm 7^{\mathrm{ab}}$ & $69 \pm 4^{\mathrm{b}}$ \\
\hline
\end{tabular}

All values are given as mean \pm SEM, $n=10$. Erythema is scored on a 0-9 scale and, except for epidermal thickness and mitotic figures, all other parameters are scored on a 0-4 scale. For each parameter, pairwise comparisons of means with at least one superscript in common denote nonsignificant differences at the $0 \cdot 05$ level.
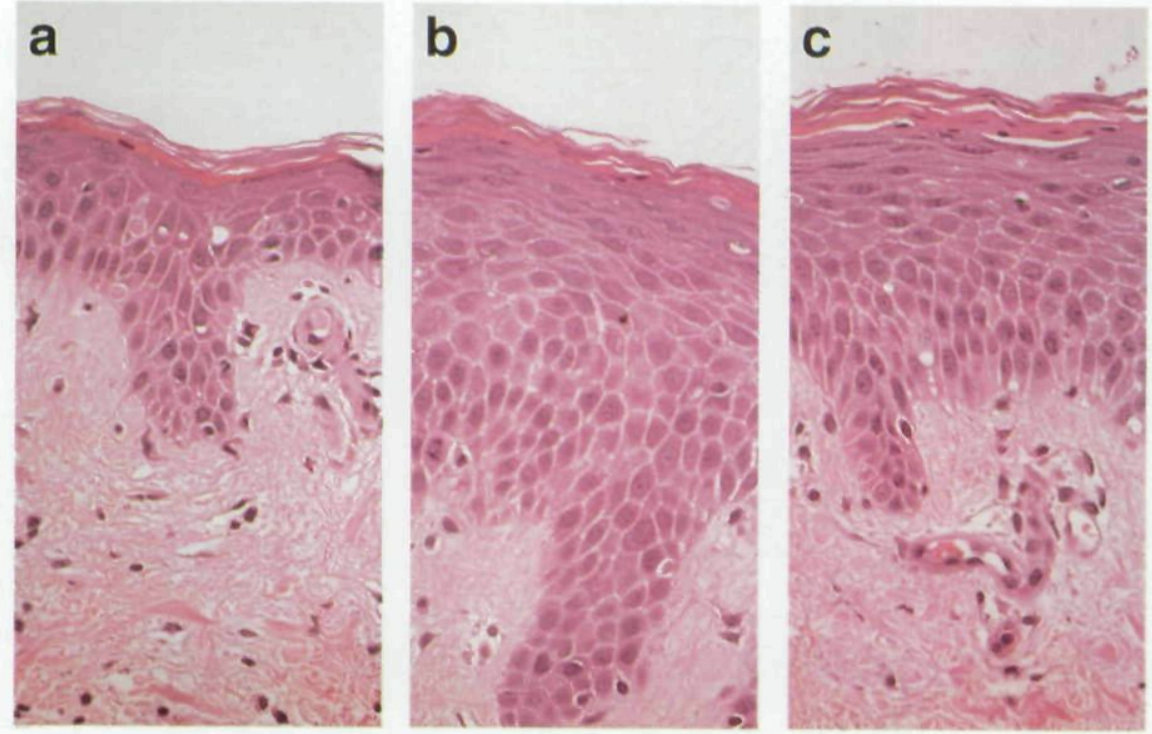

\begin{tabular}{lcccc}
\hline & $0 \cdot 1 \% \mathrm{RA}$ & $0 \cdot 025 \% \mathrm{RA}$ & $2 \%$ SLS & Vehicle \\
\hline Erythema & $3 \cdot 6 \pm 0 \cdot 5^{\mathrm{a}}$ & $3 \cdot 0 \pm 0 \cdot 4^{\mathrm{a}}$ & $4 \cdot 6 \pm 0 \cdot 8^{\mathrm{a}}$ & $0 \cdot 7 \pm 0 \cdot 4^{\mathrm{b}}$ \\
Stratum corneum compaction & $2 \cdot 2 \pm 0 \cdot 4^{\mathrm{ab}}$ & $2 \cdot 6 \pm 0 \cdot 3^{\mathrm{ab}}$ & $3 \cdot 4 \pm 0 \cdot 5^{\mathrm{a}}$ & $1 \cdot 4 \pm 0 \cdot 5^{\mathrm{b}}$ \\
Granular layer thickness & $1 \cdot 4 \pm 0 \cdot 3^{\mathrm{a}}$ & $1 \cdot 3 \pm 0 \cdot 2^{\mathrm{a}}$ & $1 \cdot 4 \pm 0 \cdot 2^{\mathrm{a}}$ & $0 \cdot 8 \pm 0 \cdot 1^{\mathrm{a}}$ \\
Spongiosis & $2 \cdot 7 \pm 0 \cdot 3^{\mathrm{a}}$ & $2 \cdot 8 \pm 0 \cdot 4^{\mathrm{a}}$ & $1 \cdot 0 \pm 0 \cdot 4^{\mathrm{b}}$ & $0 \cdot 5 \pm 0^{\mathrm{b}}$ \\
Mitoses $/ 5$ h.p.f. & $1 \cdot 0 \pm 0 \cdot 2^{\mathrm{a}}$ & $0 \cdot 7 \pm 0 \cdot 3^{\mathrm{a}}$ & $0 \cdot 4 \pm 0 \cdot 2^{\mathrm{ab}}$ & $0 \cdot 0 \pm 0^{\mathrm{b}}$ \\
Epidermal thickness $(\mu \mathrm{m})$ & $105 \pm 9^{\mathrm{a}}$ & $95 \pm 6^{\mathrm{a}}$ & $94 \pm 8^{\mathrm{a}}$ & $61 \pm 5^{\mathrm{b}}$ \\
\hline
\end{tabular}

All values are given as mean \pm SEM, $n=7$. Erythema is scored on a 0-9 scale and, except for epidermal thickness and mitotic figures, all other parameters are scored on a $0-4$ scale. For each parameter, pairwise comparisons of means with at least one superscript in common denote nonsignificant differences at the 0.05 level. 

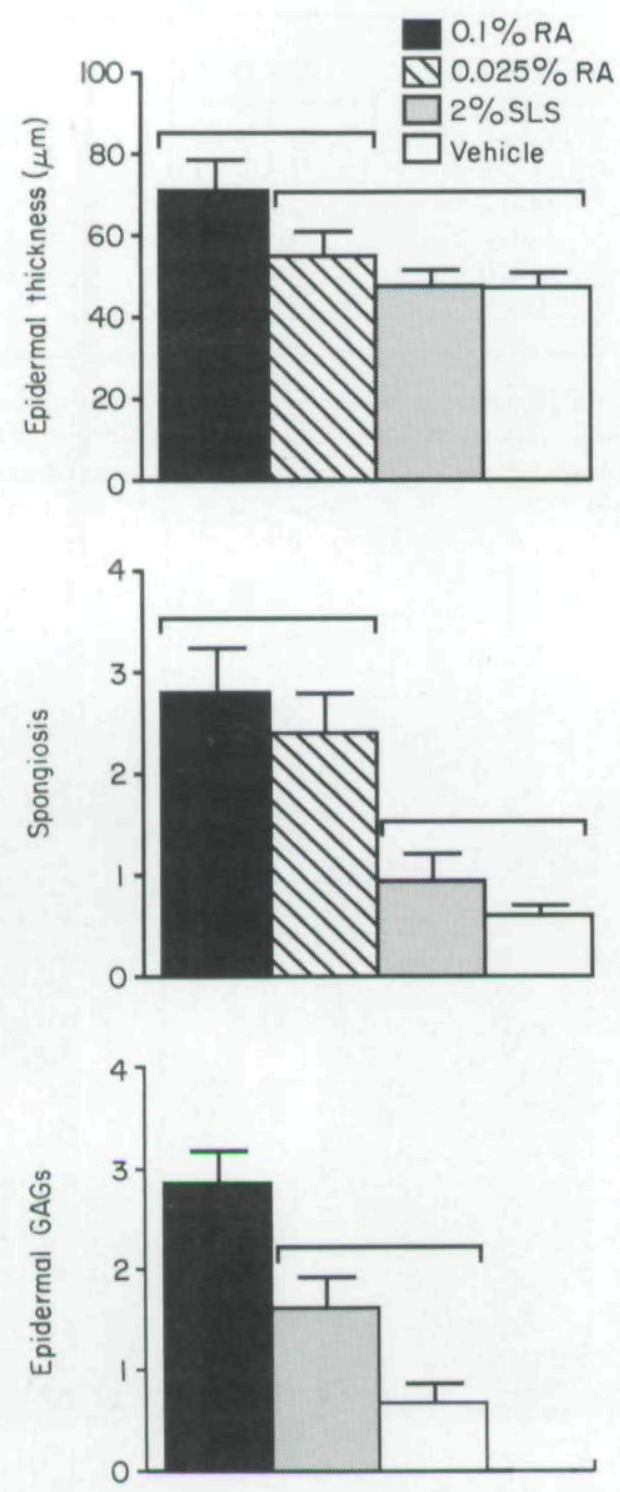

Figure 2. Skin treated for 2 days under occlusion with $0 \cdot 1 \%$ RA. $0 \cdot 025 \%$ RA, $2 \%$ SLS and vehicle creams. Spongiosis and epidermal GAGs measured on a 0-4 ordinal scale; epidermal thickness was assessed in 5 high-power fields and the mean value used. All values are mean \pm SEM. Horizontal lines span bars which are not significantly different at the 0.05 level.

were statistically indistinguishable from each other. $2 \%$ SLS produced less spongiosis than either $0 \cdot 1 \%$ or $0.025 \%$ RA, but was not significantly different from RA for all other histological features (Table 2).

Two days. At 2 days, although no erythema was seen in any of the vehicle-treated sites, there was a range of $0-4$ for $0.1 \%$ RA and SLS and $0-2$ for $0.025 \%$ RA. Consequently, with this wide range of response the only significant difference in erythema was between $2 \%$ SLS and vehicle $(P<0 \cdot 05)$.
The 2-day test was unable to reliably differentiate the two concentrations of RA from one another, but readily distinguished $0 \cdot 1 \%$ RA from SLS by virtue of increased epidermal thickness, spongiosis and epidermal GAG content in RA-treated skin (Fig. 2). GAGs were not measured in $0 \cdot 025 \%$ RA-treated specimens.

One day. Neither SLS nor vehicle produced erythema at 1 day. However, there was an erythema score of 1 for both $0 \cdot 025 \%$ and $0 \cdot 1 \%$ RA in two subjects. SLS $(0 \cdot 8 \pm 0 \cdot 3)$ produced significantly more stratum corneum compaction than vehicle $(0 \cdot 1 \pm 0 \cdot 1)$ or $0 \cdot 025 \%$ RA $(0 \pm 0$, $P<0.05$ and 0.01 , respectively). There were no other significant differences between the agents at this timepoint.

\section{Dose-response of RA in ETOH/PG vehicle}

A dose-response was generated from 0.001 to $0.025 \%$ RA, using erythema, epidermal thickness and spongiosis (Table 3). Epidermal thickness was the best discriminating parameter (Fig. 3).

\section{Discussion}

These studies represent a confirmation of the fidelity of the 4-day patch assay as a means of demonstrating an epidermal retinoid effect using RA. In addition, we have demonstrated that at early time points, i.e. 2 days, $2 \%$ SLS can be reliably differentiated from $0 \cdot 1 \%$ RA with regard to epidermal hyperplasia, spongiosis, and deposition of GAG. The assay has sufficient discriminatory power to provide a dose-response for RA in ETOH-PG vehicle.

The 4-day assay is primarily a measure of epidermal retinoid effects, and to date we have little evidence that dermal components, i.e. extracellular matrix, are altered within such a short time-frame (C.E.M. Griffiths and J.J. Voorhees, unpublished observations). However, we believe that in vivo dermal changes would parallel epidermal events, and that the observed histological changes in the epidermis predict later, slow-onset biological activity within the dermis.

$2 \%$ SLS compounded in Retin- $\mathrm{A}^{\mathrm{TM}}$ vehicle produces clinical and epidermal histological effects virtually indistinguishable from those of $0 \cdot 1 \% \mathrm{RA}$ in the 4 -day assay. ${ }^{8}$ the only difference at 4 days being an increased deposition of GAG in RA-treated epidermis. ${ }^{17}$ The 2-day assay is able to distinguish between $0 \cdot 1 \% \mathrm{RA}$ and $2 \%$ SLS, in that RA produces significant increases in epidermal thickness, spongiosis, and epidermal GAG when com- 
Table 3. 4-day patch test: clinical and histological parameters for $0 \cdot 001-0 \cdot 025 \%$ RA in ETOH/PG vehicle

\begin{tabular}{|c|c|c|c|c|c|c|}
\hline & $0 \cdot 025 \% \mathrm{RA}$ & $0 \cdot 01 \%$ RA & $0 \cdot 005 \%$ RA & $0.0025 \%$ RA & $0.001 \%$ RA & Vehicle \\
\hline Erythema & $4 \cdot 2 \pm 0 \cdot 2^{\mathrm{a}}$ & $2 \cdot 7 \pm 0 \cdot 2^{b}$ & $2 \cdot 8 \pm 0 \cdot 2^{b}$ & $2 \cdot 4 \pm 0 \cdot 2^{\mathrm{bc}}$ & $1 \cdot 9 \pm 0 \cdot 2^{b c}$ & $1 \cdot 5 \pm 0 \cdot 2^{c}$ \\
\hline Stratum corneum compaction & $0 \cdot 9 \pm 0 \cdot 1^{a b}$ & $1 \cdot 0 \pm 0 \cdot 1^{\mathrm{ab}}$ & $1 \cdot 3 \pm 0 \cdot 2^{b}$ & $1 \cdot 1 \pm 0 \cdot 1^{\mathrm{b}}$ & $0 \cdot 4 \pm 0 \cdot 1^{\mathrm{a}}$ & $0 \cdot 7 \pm 0 \cdot 2^{\text {ab }}$ \\
\hline Granular layer thickness & $1 \cdot 9 \pm 0 \cdot 2^{\mathrm{a}}$ & $1 \cdot 9 \pm 0 \cdot 2^{\mathrm{a}}$ & $2 \cdot 1 \pm 0 \cdot 2^{\mathrm{a}}$ & $1 \cdot 7 \pm 0 \cdot 2^{a}$ & $0 \cdot 9 \pm 0 \cdot 1^{b}$ & $0 \cdot 6 \pm 0 \cdot 1^{b}$ \\
\hline Spongiosis & $2 \cdot 9 \pm 0 \cdot 1^{\mathrm{a}}$ & $2 \cdot 5 \pm 0 \cdot 1^{\text {ac }}$ & $2 \cdot 4 \pm 0 \cdot 2^{\text {ad }}$ & $2 \cdot 2 \pm 0 \cdot 2^{\mathrm{cd}}$ & $1 \cdot 2 \pm 0 \cdot 1^{b}$ & $0 \cdot 9 \pm 0 \cdot 1^{b}$ \\
\hline Mitoses $/ 5$ h.p.f. & $0 \cdot 4 \pm 0 \cdot 1^{a}$ & $0 \cdot 2 \pm 0 \cdot 1^{\mathrm{a}}$ & $0 \cdot 2 \pm 0 \cdot 1^{\text {a }}$ & $0 \cdot 4 \pm 0 \cdot 2^{a}$ & $0.03 \pm 0.03^{a}$ & $0.03 \pm 0.03^{a}$ \\
\hline Epidermal thickness $(\mu \mathrm{m})$ & $89 \pm 3^{a}$ & $79 \pm 3^{\mathrm{ac}}$ & $79 \pm 3^{\text {ac }}$ & $73 \pm 3^{b c}$ & $56 \pm 2^{\mathrm{d}}$ & $53 \pm 3^{\text {d }}$ \\
\hline
\end{tabular}

All values are given as mean \pm SEM, $n=30$. Erythema is scored on a $0-9$ scale and, except for epidermal thickness and mitotic figures, all other parameters are scored on a $0-4$ scale. For each parameter, pairwise comparisons of means with at least one superscript in common denote nonsignificant differences at the $0 \cdot 05$ level.

pared with vehicle, whereas SLS is indistinguishable from vehicle for these parameters. Although cutaneous application of RA and SLS in this assay may produce similar epidermal histological phenotypes, it is likely that this end-point is achieved differently by the two agents.

RA-mediated effects are probably receptor-dependent, and achievable by direct interactions with nuclear RA receptors ${ }^{18-22}$ and specific binding proteins, namely cellular retinoic acid binding protein (CRABP). ${ }^{23}$ It has been demonstrated that RA but not SLS in the occlusion assay will increase CRABP-II mRNA, implying that CRABP-II is a sensitive, and perhaps an RA-specific, marker. ${ }^{24}$ SLS, like RA, can directly stimulate proliferation of keratinocytes and fibroblasts in vitro, but does not stimulate a reporter gene harbouring a retinoic acid responsive element in cells co-transfected with the reporter construct and a construct that will endow the cell with RA receptors. ${ }^{25}$ Thus, receptor-dependent

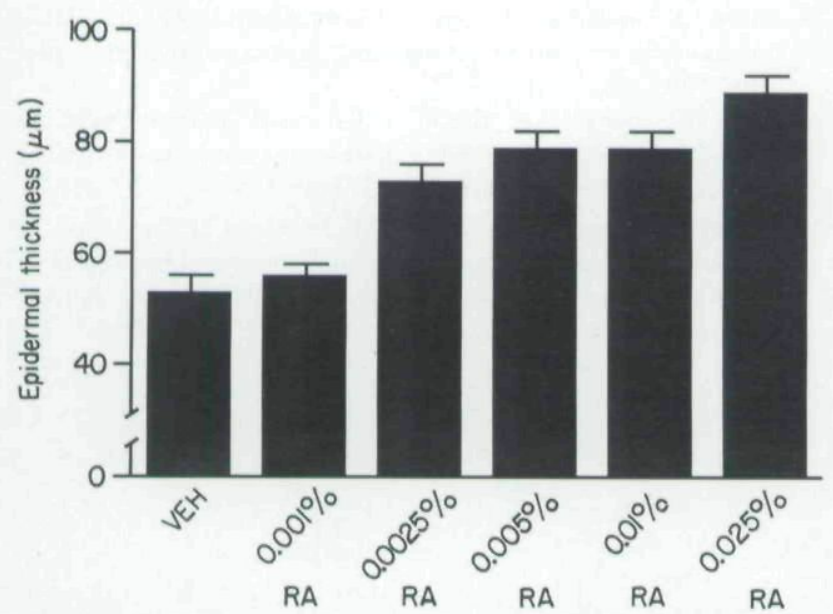

Figure 3. Epidermal thickness of skin treated for 4 days under occlusion with different concentrations of RA in ETOH/PG vehicle or vehicle alone. All values are mean \pm SEM. events most likely lead to release of growth factors such as transforming growth factor- $\beta_{1},{ }^{17}$ which may determine the characteristic 'retinoid' epidermal proliferation. SLS, and perhaps other irritants, although incapable of direct interaction with RA receptors and CRABP, possibly induce release of comparable growth factors, leading to an epidermal phenotype similar to that induced by retinoids, i.e. SLS acts 'downstream' of specific receptor-mediated events and is in itself receptor independent.

This assay was unable to differentiate between $0 \cdot 1 \%$ and $0.025 \%$ RA creams, thereby implying equal potency. It is likely that much larger sample sizes may have brought out statistically significant differences between the two concentrations of RA, and allowed complete differentiation of $0.025 \%$ RA from vehicle. However, direct comparison of efficacy of $0.025 \%$ and $0 \cdot 1 \%$ RA creams in the treatment of photodamage has revealed that the two concentrations are equally efficacious in this disorder (C.E.M.Griffiths, J.J.Voorhees, unpublished observations). Alternatively, the current occlusion assay may fully maximize the effects of creamformulated topical retinoids, the peak being reached with doses at least as low as $0.025 \%$ RA cream. We have demonstrated that by dissolving RA in a relatively inert, non-cream, vehicle (ETOH-PG), a dose-response for epidermal thickness can be generated. The doses of RA can be separated into three statistically identifiable groups, namely: vehicle and 0.001\% RA: $0.0025 \%$. $0 \cdot 005 \%, 0 \cdot 01 \%$ RA; and $0 \cdot 025 \%$ RA. Epidermal thickness is a valid measure of dose-response in this assay system, as it is the only truly objective histological parameter studied. The ability to create a dose-response with RA indicates a sensitivity which could allow a grading of retinoid potency, as has been described in the hairless mouse model. ${ }^{3}$

In summary, we describe a sensitive human bioassay 
system which is capable of providing an RA potency grading, and which could be applicable to the study of comparative retinoid effects. As epidermal histological changes may predict propensity for dermal remodelling and clinical efficacy in treatment of acne and/or photodamage, this system also has potential as a predictive screening assay. Evidence is provided that although SLS produces epidermal histological changes similar to those seen with RA, these occur later, and may imply a different mode of action, i.e. receptor independent. Future work should be directed towards identifying sensitive and specific molecular targets for retinoids (perhaps CRABP-II). These target genes may allow more accurate discrimination between active and inactive moieties, and ultimately predict clinical efficacy.

\section{Acknowledgments}

This research was supported in part by the Babcock Dermatologic Endowment, Ann Arbor, MI and the R.W. Johnson Pharmaceutical Research Institute, Raritan, NJ. We are grateful to Robin Gardner for help in tissue procurement, and Dale Yessian for secretarial assistance.

\section{References}

1 Kligman LH, Kligman AM. The effect on rhino mouse skin of agents which influence keratinization and exfoliation. I Invest Dermatol 1979: 73: 354-8.

2 Ashton RE, Connor MJ, Lowe NJ. Histologic changes in the skin of rhino mouse $\left(h r^{\text {rh }} h r^{r h}\right)$ induced by retinoids. I Invest Dermatol 1984: 82: 632-5.

3 Connor MJ. Ashton RE, Lowe NJ. A comparative study of the induction of epidermal hyperplasia by natural and synthetic retinoids. J Pharmacol Exp Ther 1986; 237: 31-5.

4 Kligman LH. Effects of all-trans-retinoic acid on the dermis of hairless mice. J Am Acad Dermatol 1986: 15: 779-85.

5 Boucher M, Chatelus A. Ferracin Jet al. Quantification of epidermal histological changes induced by topical retinoids and $\mathrm{CD} 271$ in the rhino mouse model using a standardized image analysis technique. Skin Pharmacol 1991: 4: 65-73.

6 Varani J. Gendimenico GJ. Shah B et al. A direct comparison of pharmacologic effects of retinoids on skin cells in vitro and in vivo. Skin Pharmacol 1991; 4: 254-61.

7 Griffiths CEM, Rosenthal DS, Reddy AP et al. Short-term retinoic acid treatment increases in vivo, but decreases in vitro, epidermal
transglutaminase-K enzyme activity and immunoreactivity. I Invest Dermatol 1992; 99: 283-8.

8 Fisher GJ, Esmann J, Griffiths CEM et al. Cellular, immunologic and biochemical characterization of topical retinoic acid-treated human skin. J Invest Dermatol 1991: 96: 699-707.

9 Weiss JS, Ellis CN. Headington JT et al. Topical tretinoin improves photoaged skin. A double-blind, vehicle-controlled study. JAMA 1988; 259: 527-32.

10 Bhawan J, Gonzalez-Serva A, Nehal K et al. Effects of tretinoin on photodamaged skin. A histologic study. Arch Dermatol 1991: 127: 666-72.

11 Rafal ES, Griffiths CEM, Ditre CM et al. Topical tretinoin (retinoic acid) treatment for liver spots associated with photodamage. $\mathrm{N}$ Engl J Med 1992; 326: 368-74.

12 Kligman AM, Grove GL, Hirose R, Leyden JJ. Topical tretinoin for photoaged skin. J Am Acad Dermatol 1990; 15: 836-59.

13 Lever L, Kumar P, Marks R. Topical retinoic acid for the treatment of solar damage. Br J Dermatol 1990; 122: 91-8.

14 Caputo R, Monti M, Motta S et al. The treatment of visible signs of senescence: the Italian experience. Br J Dermatol 1990: 122 (Suppl. 35): 97-103.

15 Weinstein GD, Nigra TP, Pochi PE et al. Topical tretinoin for treatment of photodamaged skin. A multicenter study. Arch Dermatol 1991; 127: 659-65.

16 Olsen EA, Katz I, Levine N et al. Tretinoin emollient cream: a new therapy for photodamaged skin. J Am Acad Dermatol 1992; 26: 215-24.

17 Fisher GJ, Tavakkol A, Griffiths CEM et al. Differential modulation of transforming growth factor-beta 1 expression and mucin deposition by retinoic acid and sodium lauryl sulfate in human skin. J Invest Dermatol 1992; 98: 102-8.

18 Petkovich M, Brand NJ, Krust A, Chambon P. A human retinoic acid receptor which belongs to the family of nuclear receptors. Nature 1987: 330: 444-50.

19 Brand N, Petkovich M, Krust A. et al. Identification of a second human retinoic acid receptor. Nature 1988; 332: 850-3.

20 Krust A, Kastner P, Petkovich M et al. A third human retinoic acid receptor hRAR- $\gamma$. Proc Natl Acad Sci USA 1989; 86: 5310-14.

21 Elder JT, Fisher GJ, Zhang QY et al. Retinoic acid receptor gene expression in human skin. J Invest Dermatol 1991: 96: 425-33.

22 Rees JL, Redfern CPF. Expression of the $\alpha$ and $\beta$ retinoic acid receptors in skin. J Invest Dermatol 1989: 92: 818-20.

23 Astrom A, Tavakkol A, Pettersson U et al. Molecular cloning of two human cellular retinoic acid-binding proteins (CRABP). $J$ Biol Chem 1991; 266: 7662-6.

24 Elder JT. Cromie MA. Griffiths CEM et al. Stimulus-selective induction of CRABP-II mRNA: a marker for retinoic acid action in human skin. J Invest Dermatol 1993: 100: 356-9.

25 Varani J. Astrom A, Griffiths CEM, Voorhees JJ. Induction of proliferation of growth-inhibited keratinocytes and fibroblasts in monolayer culture by sodium lauryl sulfate: comparison with alltrans retinoic acid. J Invest Dermatol 1991; 97: 917-21. 
This document is a scanned copy of a printed document. No warranty is given about the accuracy of the copy. Users should refer to the original published version of the material. 